\title{
METODOLOGIAS PARA INCORPORAR EL \\ DIALOGO DE SABERES EN LA FORMACIÓN \\ SUPERIOR DE DOCENTES DE PUEBLOS \\ INDIGENAS
}

Ingrid Guzmán Sota ${ }^{1}$

\section{RESUMO}

$\mathrm{O}$ presente artigo discute possibilidades de metodologias interessadas em, por meio do diálogo intercultural, incorporar os saberes ancestrais dos povos originários nos cursos de formação superior de professores indígenas, visto que essa é uma antiga demanda dos povos indígenas da América Latina. A incorporação desses saberes ancestrais garante a permanência de uma diversidade cultural que permite a adoção de uma pedagogia intercultural, o que contribui para a formação de docentes indígenas que efetivamente produzem ações que perpetuam esses saberes e inovam nas metodologias de ensino das escolas indígenas.

PALAVRAS-CHAVE: Saberes ancestrais. Metodologias de ensino. Formação superior indígena.

\section{RESÚMEN}

El presente artículo discute posibilidades de metodologías interesadas en, a través del diálogo intercultural, incorporar los saberes ancestrales de los pueblos originarios en los cursos de formación superior de profesores indígenas, ya que esa es una antigua demanda de los pueblos indígenas de América Latina. La incorporación de esos saberes ancestrales garantiza la permanencia de una diversidad cultural que permite la adopción de una pedagogía intercultural, lo que contribuye a la formación de docentes indígenas que efectivamente producen acciones que perpetúan esos saberes e innovan en las metodologías de enseñanza de las escuelas indígenas.

PALABRAS CLAVE: Saberes ancestrales. Metodologías de enseñanza. Formación superior indígena.

1 Professora da Pontificia Universidad Católica Del Perú. Lima, Peru.

E-mail: ingridweber@mec.gov.br.' 


\section{ABSTRACT}

The present article discusses possibilities of methodologies interested in, through intercultural dialogue, to incorporate the ancestral knowledge of the native peoples in the courses of superior formation of indigenous teachers, since this is an old demand of the indigenous peoples of Latin America. The incorporation of these ancestral knowledge guarantees the permanence of a cultural diversity that allows the adoption of an intercultural pedagogy, which contributes to the formation of indigenous teachers who effectively produce actions that perpetuate these knowledge and innovate in the teaching methodologies of indigenous schools.

KEYWORDS: Ancestral knowledge. Teaching methodologies. Indigenous higher education.

\section{Acercamiento Conceptual}

Respecto al diálogo de saberes en los últimos cincuenta años se han desarrollado múltiples experiencias en el marco de los procesos de la educación popular y comunitaria, y la reflexión al respecto se remonta a los años 60 y 70 , cuando desde la investigación acción participativa se propone un diálogo de saberes, "en ese momento referido al encuentro entre un mundo académico en crisis y unos actores sociales que surgían con fuerza a nivel global, pero especialmente en los países en proceso de descolonización" (ARCHILA, 2017). Sobre la conceptualización del diálogo de saberes asumimos que“...constituye la relación mutuamente enriquecedora entre personas y culturas, puestas en colaboración por un destino compartido" (ISHIZAWA, 2016).

Desde la vida de los pueblos originarios en Latinoamérica, el diálogo de saberes constituye una demanda sentida y una práctica vivida de diversidad cultural. Aprendemos de ellos que para mantener la vida sobre la base de la biodiversidad "se requiere de ambos saberes o forma de conocimiento, el occidental moderno que se enseña en la escuela y el propio que surge como componente esencial de un modo de vida distinto, se consideren como pares o en una relación de equivalencia." (ISHIZAWA, 2016). 
Desde la mirada de estudiosos del desarrollo como Thomas Berry “...El diálogo de saberes es una vía de comprensión de los dilemas que plantea la crisis ecológica, como resultado del proyecto globalizador de dominación, que debe traducirse en soluciones que se complementan y orientan estrategias y acciones conjuntas; dado que ninguna cultura, en particular, posee la sabiduría necesaria para revertir sola los efectos negativos del desarrollo de las últimas décadas" (BERRY, 1999)

Berry destaca la cuádruple sabiduría que constituye la fuente de conocimiento que podría contribuir a enfrentar los retos presentes:

1. La sabiduría indígena que se distingue por "su intimidad con, y participación en, el funcionamiento del mundo natural".

2. La sabiduría de las mujeres que consiste en "unir el conocimiento del cuerpo con el de la mente, reunir alma y espíritu, intuición a razonamiento, conciencia sintiente y análisis intelectual, intimidad y desapego, presencia subjetiva y distancia objetiva".

3. La sabiduría de las tradiciones clásicas (hindú, china, occidental, griega, bíblica, romana, [...]) basada en las experiencias de un dominio espiritual, trascendentes e inmanentes en un mundo visible en torno nuestro, y en la capacidad de los humanos, de participar en ese mundo para lograr la completitud de su propio modo de ser".

4. La sabiduría de la ciencia que "devela el proceso del universo como una secuencia de transformaciones en un inmenso período temporal. A través de estos episodios de transformación, el universo ha pasado de una menor a una mayor complejidad en estructura y de un menor a un mayor modo de conciencia... esta conciencia es el gran aporte de la ciencia occidental moderna".

En último término, el diálogo de saberes apuntaría a la convergencia de esta cuádruple sabiduría para orientar un vivir humano en armónica relación con la cosmogénesis que es el universo, siempre y cuando se genere condiciones para una equivalencia de las culturas y cosmovisiones puestas en juego en el diálogo y el reconocimiento mutuo de la intrínseca legitimidad de los participantes.

En el marco de la política nacional EIB y del nuevo currículum para la formación de docentes EIB en Institutos Pedagógicos de Perú se precisa que el diálogo de saberes se entiende: "como un proceso de 
interrelación dinámica, enriquecedora y permanente entre sistemas de conocimientos de diferentes tradiciones culturales, que se aborda pedagógicamente y permite responder a problemáticas y desafíos locales y globales. Busca superar la concepción de la existencia de un único medio válido -universal- de interpretar la realidad y actuar en ella." (Ministerio de Educación de Perú: DIFOIB- DEIB, 2017).

Una aproximación a los procesos didácticos de diálogo de saberes debería recoger las formas de concreción señaladas por el ministerio de educación, pero su dimensión didáctica deberá expresarse en:

- Organización de los saberes locales

- Investigación de saberes de otros

- Síntesis y diseño de prácticas transformadoras.

Lo que implicaría que el docente formador asuma un rol de acompañante intercultural y que desarrolle los procesos didácticos acompañado de expertos en la sabiduría local, que pueden encontrarse entre los mismos alumnos o en sus pueblos de origen: este acompañante/formador tendría que:

1. Saber escuchar la sabiduría de los abuelos, poniendo en suspenso sus habilidades profesionales y su pretendida autoridad cognitiva en su relación con los que acompaña (desprofesionalización).

2. Saber desaprender, es decir, saber poner en cuestión lo que da por sentado para reflexionarlo con los colegas y con los que acompaña (descolonización).

3. Tener conciencia de que se mueve constantemente entre cosmovisiones radicalmente distintas y que esta situación exige saber en cada situación desde qué cosmovisión se encuentra actuando y cuáles son las consecuencias de las elecciones conscientes o inconscientes que está haciendo (ISHIZAWA, 2016). 


\section{Un Acercamiento desde la Experiencia}

\section{La metodología general del diálogo de saberes}

En la formación de docentes EIB la pedagogía intercultural se constituye en el marco orientador para construir procesos de afirmación identitaria de los estudiantes en formación, la identificación de formas diversas de discriminación, el análisis de sus causas y consecuencias y la construcción de mecanismos para afrontarla; el reconocimiento y valoración de diversas formas de entender la realidad y de distintas maneras de construir el conocimiento y de aprender; y la toma de conciencia de la existencia de derechos individuales y colectivos y la reflexión sobre el ejercicio de los mismos, en esencia en este proceso se va construyendo un diálogo intercultural que sigue más o menos este proceso:

5. Acciones de visibilización del aporte del saber originario

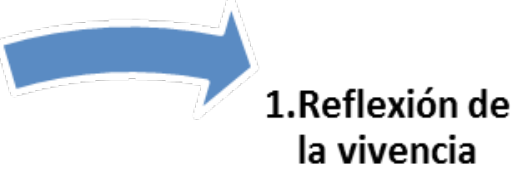

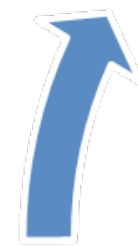

4. Conclusiones

que orientan la acción
2.Sistematización y desarrollo de saberes sobre la vivencia

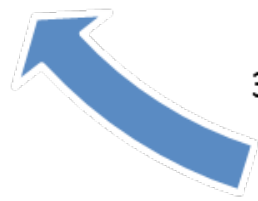

3. Comprensión de los saberes de otros pueblos originarios y de la ciencia 
Esta configuración de la pedagogía intercultural no puede solo teorizarse, abordarse conceptualmente en la formación de los futuros docentes, sino que debe reconstruirse vivencialmente desde la propia historia de aprendizajes, permeada muchas veces por experiencias poco estimulantes, con dolorosas situaciones de ruptura y vergüenza social, sobre todo cuando se refiere al espacio escolar: cuando era pequeña el maestro nos amenazaba con la evaluación, si no contestábamos igual que lo que decía el profesor no valia, me sentía inútil, que no valia (Estudiante 1:2017).

El análisis de la vivencia permite identificar en la experiencia de socialización cultural procesos educativos legítimos y valiosos para la conformación del ser docente, por ejemplo en el campo de la evaluación educativa, a modo de contraste con la experiencia escolar, un estudiante menciona:

No me había dado cuenta que las ferias de mi distrito eran espacios para evaluar lo que las familias saben y hacen, además allí ninguna familia vale más, todas son necesarias, las que cultivan maíz, las que hacen chuño (papa deshidratada al frio) las que hacen charki(carne de llama o alpaca seca salada, cada uno tiene su sabiduría, lo importante es que tengan un buen producto para que intercambien bien con otros....así debería ser la evaluación en la escuela (Estudiante 2:2017).

La reflexión de la vivencia apertura un espacio para revaluar desde una dimensión crítica reflexiva la experiencia escolar y alimentar desde la revitalización de la sabiduria comunitaria formas alternativas de evaluación dentro de la pedagogía intercultural en este caso.

La reconstrucción de una vivencia puede cuestionar y poner en tensión los valores y prácticas comunitarias también:

Ser duros y exigentes con los hijos es parte de la vida de la comunidad...yo me sentía mal, nunca estuvo para apoyarme mi padre, no era bien visto, era como ser débil....y a mi en la escuela me hacían aparte... pero él no me apoyaba...más me desvalorizaba" "Pero yo aquí en dialogo con muchas personas he aprendido a quererme y valorarme como persona (Estudiante 3: 2017). 
Este testimonio nos da cuenta del cuestionamiento de los roles asignados en la pervivencia familiar a varones y mujeres, los cuales se socializan en un escenario de exigencia, dureza, y distancia afectiva; para el caso de muchas familias, en esta cultura originaria. Estas pautas de relacionamiento se redimensionan en los espacios urbanos, incorporando la noción y el valor del derecho individual y la equidad en la relaciones entre individuos.

La pregunta es ¿cómo se aborda la vivencia en el proceso de diálogo de saberes?. En la experiencia desarrollada es siempre el momento que apertura el abordaje de un tema o noción, la vivencia pasada se recupera desde una situación de aprendizaje afectiva que te pone en dialogo con un par, otro joven varón o mujer que escucha y también comparte, espacio que adicionalmente sirve para tejer lazos de cercanía y confianza, pese a las diferencias culturales, de género y de edad.

Es preciso preguntarse si la vivencia individual es suficiente para recomponer los vínculos con la cultura y hacer consciente la racionalidad que subyace a los saberes locales?... sin duda es insuficiente, se requiere recuperar los significados profundos de estas sabidurías desde diferentes voces, entretejiendo las experiencias y las reflexiones e identificando organizadores de información que permitan expresar en su real dimensión el aprendizaje, es preciso entonces abrirse al diálogo con la comunidad y sus actores: los ancianos, las mujeres, los especialistas en diferentes sabidurías, para escuchar y asumir el desafío de organizar esta información para hacerla comunicable a otros.

La experiencia muestra que los organizadores gráficos expresan mejor el universo de significados de la cosmovisión de los pueblos originarios, su belleza e integralidad para plasmar articuladamente las comprensiones del mundo, es reconocida desde el tiempo de la invasión y colonización y es un bien cultural que trasciende de generación en generación.

Sin embargo no importa solo la dimensión expresiva en este momento, es clave además mirar los saberes en contexto, y dar cuenta de su condición de subordinación, de los desafíos que se enfrenta por las limitaciones en su propia estructura por pérdida o desuso, fruto de un desarrollo cultural impuesto y marginalizador de todas las expresiones de los pueblos originarios o asimilador de su dinámica para beneficio propio. Sin duda surgen desafíos que comprometen a los jóvenes con la reproducción cultural situada y 
crítica, en el desafío de hacerse visibles desde la especificidad de su sabiduría.

Los y las jóvenes requieren además incorporar otras visiones sobre los hechos o conceptos que comprometen su formación y acercarse a aproximaciones de otros pueblos originarios sobre los mismos hechos, para empezar a tejer solidaridades y reconocerse como parte de una colectividad mayor capaz de desarrollar una fuerza que luche por la hegemonía del poder desde el conocimiento compartido que maneja.

Pero es también importante dialogar con visiones teóricas/ prácticas construidas desde la ciencia positiva o interpretativa, menuda tarea que reviste una complejidad mayor dadas las deficiencias en su formación básica donde con frecuencia fueron compulsivamente obligados a apropiarse de la lectoescritura en otra lengua que no era su lengua materna. La lectura recreativa ayuda a hacer más amable este proceso, los organizadores de ideas expresados en mapas mentales donde se aprecian la relación entre conceptos son también claves, porque precisamente se enganchan con la visión totalizadora del mundo de nuestros pueblos originarios.

Un soporte valioso para la comprensión de contenidos teórico/ prácticas de las ciencias pedagógicas es su redimensionamiento a través de medios expresivos más lúdicos y amigables como la creación de canciones, teatralizaciones, poesías, murales. Comprendidos los contenidos desde estas otras perspectivas recién los jóvenes de pueblos originarios pueden una postura personal frente a ellos, hacerla entrar en diálogo con el saber originario y otorgarle validez para afrontar los problemas sociales y educativos.

Desde la visión de los pueblos originarios un aprendizaje es efectivo solamente si es práctico, es decir ayuda a resolver un problema del contexto, si transforma el ser personal pero además el espacio colectivo y aporta a diseñar herramientas concretas de actuación: una sesión de clase innovadora que aporta a la convivencia intercultural, un mural que da cuenta de la visión educativa de los pueblos originarios, una intervención educativa en escuelas urbanas para que comprendan y respeten las cosmovisiones andinas, una campaña en la universidad para interculturalizar las relaciones, los enfoques y las miradas de formación, todas esas acciones son aplicaciones concretas y visibles de lo aprendido.

La presencia pública a través de los productos de sus aprendizajes, poniendo en evidencia el valor de los saberes de los 
pueblos originarios y sus posibilidades de ser sistemáticos, científicos y de constituirse en referente formativo en los diferentes campos del conocimiento ha devuelto orgullo, identidad y afirmación a los estudiantes de educación intercultural bilingüe de estos pueblos. Es ese el corolario de un proceso que no solo es pedagógico sino político.

\section{Orientaciones para la Acción}

Este proceso construido en acciones de investigación acción participativa con los estudiantes e la carrera de Educación Intercultural Bilingüe, nos lanza a desafíos mayores que debemos sopesar de manera colectiva, quienes estamos interesados y comprometidos con hacer de la formación superior en las Universidades un escenario de equidad en la diversidad y de convivencia intercultural:

- La viabilidad de construir un diálogo de saberes en la formación pedagógica, reside en la acción de centrar la enseñanzaaprendizaje en la comprensión y solución de los problemas de inequidad existentes en el servicio educativo en desmedro de las poblaciones originarias, así como la conflictiva relación entre escuela y comunidad para diseñar soluciones originales reconociendo el aporte de los saberes locales, saberes de otros pueblos y de la ciencia positiva.

- Incorporar los discursos y cosmovisiones de los pueblos originarios en la comprensión de la ciencia pedagógica es una condición necesaria para devolverle legitimidad a la pedagogía comunitaria y establecer una pluralidad académica en la formación de los jóvenes.

- Se precisa descolonizar la forma de relación entre el conocimiento académico dominante y las comprensiones del mundo de las poblaciones originarias, repensando las exclusiones y prejuicios a través del fortalecimiento del protagonismo de otros actores sociales en la educación superior, como los propios alumnos y los especialistas de sus comunidades, portadores todos ellos de su sabiduría ancestral.

- Utilizar formas diversas de cifrar la comprensión y construcción del conocimiento, que no pase solo por el 
recurso de la lectura y escritura académica, aprovechando los recursos ancestrales de la oralidad, la representación iconográfica y los recursos audiovisuales.

- Incentivar la investigación en diversos paradigmas (cuanti y cualitativos) desarrollando los saberes en función de su utilidad para enfrentar los grandes problemas que enfrenta en la actualidad la educación intercultural bilingüe en diversos escenarios de desarrollo y revitalización.

- Generar espacios de resolución efectiva de conflictos entre diversos a partir de la participación activa de los estudiantes, centrada en el objetivo de articular soluciones dialogadas a las situaciones de discriminación y exclusión.

\section{Bibliografía}

AGUADO, T. (2003). La pedagogía intercultural. Madrid: S.A. MCGRAW-HILL / INTERAMERICANA DE ESPAÑA.

ARCHILA, M. (2017). ¿Cómo entender el diálogo de saberes? ON LASA 2017, 61-62 pp.

BERRY, T. (1999). The Great Work: Our Way in to The Future. New York - USA: Harmony/Bell Tower.

EDWARS, V. (1995). Los sujetos y la construcción social del conocimiento escolar en primaria. Santiago de Chile: IIPE.

HUBERMAN, S. (1996). Como Aprenden los que Enseñan. Buenos Aires - Argentina: Aique.

ISHIZAWA, J. (2016). Comunidades Epistémicas para el diálogo de saberes. En Ciencias, diálogo de saberes y transdisciplinaridad. Bolivia: Plural Editores.

MERINO, J. Y. (mayo - agosto de 1995). Ejes de debate y propuestas de acción para una pedagogía. Madrid, Madrid, España.

Ministerio de Educación de Perú: DIFOIB- DEIB. (2017). EL CURRICULO DE FORMACIÓN EN EDUCACIÓN INICIAL Y PRIMARIA. Lima: MINEDU. 